\title{
19th International Stirling Engine Conference
}

\author{
Luca Cioccolanti ${ }^{1}$ \\ ${ }^{1}$ Technical Secretary of Stirling International Association
}

International Stirling Engine Conference (ISEC) series has a long history. It has begun in Great Britain in 1982 and it takes place each other year with few exceptions. ISEC puts together distinguished professors and researchers from industry and academia all over the world and aims at discussing the most recent advancements on the Stirling-cycle technology.

This special volume contains contributions from the 19th ISEC, which was initially planned to be in Rome in September 2020 but later on postponed to September 2021 and held online for the prolongation of the covid-19 pandemic.

The 19th ISEC has been virtually hosted in Rome by Sapienza Università di Roma and Università eCampus thus proudly continuing the ISEC tradition. This edition of the conference series was attended by almost 70 participants from more than 20 different Countries worlwide. The conference featured more than 45 papers distributed along 8 virtual sessions.

The conference was chaired by Prof. Vincenzo Naso, Prof. Carlo Maria Bartolini and Ass. Prof. Luca Cioccolanti.

The present volume collects up-to-date contributions in the field of Stirling-cycle machines with respect to the following topics: prototypes development and testing, Stirling-cycle refrigerators and cryocoolers, Stirling heat pumps, cogeneration systems, renewable and waste heat recovery applications, transport and space applications, heat transfer/fluid flow analysis, thermal receivers, combustors and heat exchangers, regenerators and porous medium, thermodynamic modelling and CFD simulation, stress analysis/noise and vibration, dynamics and kinematics, novel design of drive mechanisms and configurations, advanced materials and manufacturing.

The volume represents an outstanding state-of-the-art of the above mentioned topics related to the Stirling-cycle technology and it provides interesting insights for current and future methodological and technological developments.

We thank the contributors and the reviewers for their outstanding work.

We wish to thank also all our colleagues of the Stirling Engine Council for their friendly support. 
We gratefully acknowledge the administrative and technical support of EIOM and, in particular, Mr Alessio Rampini, Mr Antonio Rampini and Mr Simone Casagrande. 